\title{
Bayesian Learning with Mixtures of Trees
}

\author{
Jussi Kollin and Mikko Koivisto \\ HIIT Basic Research Unit, Department of Computer Science \\ Gustaf Hällströmin katu 2b, FI-00014 University of Helsinki, Finland \\ jussi.kollin@cs.helsinki.fi, mikko.koivisto@cs.helsinki.fi
}

\begin{abstract}
We present a Bayesian method for learning mixtures of graphical models. In particular, we focus on data clustering with a treestructured model for each cluster. We use a Markov chain Monte Carlo method to draw a sample of clusterings, while the likelihood of a clustering is computed by exact averaging over the model class, including the dependency structure on the variables. Experiments on synthetic data show that this method usually outperforms the expectation-maximization algorithm by Meilă and Jordan [1] when the number of observations is small (hundreds) and the number of variables is large (dozens). We apply the method to study how much single nucleotide polymorphisms carry information about the structure of human populations.
\end{abstract}

\section{Introduction}

Mixture models provide a flexible way to learn regularities from data. By mixing simple component models one can obtain a significantly more complex model. A finite mixture can be interpreted as a semi-parametric model, with applications in density estimation and related activities. Another interpretation treats the labels of the mixture components, one per observation, as unobserved data. This latent variable formulation supports, e.g., unsupervised clustering.

Meilă and Jordan [1] present an expectation-maximization (EM) algorithm to maximum likelihood estimation of mixtures of tree-structured graphical models; remarkably, the method also estimates the tree structure. A shortcoming of this and similar maximum likelihood methods is, however, that only a single estimate of the mixture model parameters is provided, leaving the uncertainty around the estimate as poorly characterized. While bootstrapping [2] can be used for finding approximate confidence intervals, it is computationally demanding and requires a large data set. Moreover, bootstrap proportions can be hard to interpret (see, e.g., [3]) and nuisance parameters hard to handle.

Bayesian inference avoids many of the above shortcomings as it concerns the posterior distribution of the quantities of interest. Thanks to the recent developments in Markov chain Monte Carlo (MCMC) methods, Bayesian inference has become computationally feasible, at least for some important model classes, such as mixtures of multivariate Gaussians [4]. Early MCMC methods relied on Gibbs sampling that, analogously to the EM algorithm, draws samples from the joint space of model parameters and the latent component labels. Later works

J. Fürnkranz, T. Scheffer, and M. Spiliopoulou (Eds.): ECML 2006, LNAI 4212, pp. 294-305 2006.

(C) Springer-Verlag Berlin Heidelberg 2006 
(e.g., [5]) have turned to the more general Metropolis-Hastings algorithm that avoids sampling of the latent labels and is thus arguably more efficient 6]. Despite this progress, the current techniques are insufficient when the component models are more complex and involve lots of parameters [5]. This is the case, for example, when the number of variables is moderate (dozens) or large (hundreds), and when the dependency structure among the variables is unknown.

In this paper, we describe a full Bayesian method for learning with mixtures of graphical models. Our approach is motivated by two simple observations. First, for some important classes of graphical models, such as trees, there exist efficient algorithms for structure learning. Also, the space of data clusterings is discrete and "relatively small," provided that the number of data points is moderate. Consequently, it is reasonable to run MCMC over the space of clusterings, the prior and the (marginal) likelihood of a clustering being relatively cheap to evaluate. We call this approach hidden data sampling (HDS).

Related previous works have focused on simple models of independent or multivariate Gaussians. Neal [7] introduces a Markov chain sampling method for mixtures of Dirichlet processes. Rasmussen [8] describes a similar method for infinite Gaussian mixtures. Dawson and Belkhir [9], followed by Corander et al. [10, adopt the approach to a clustering problem in population genetics under a simple model where all attributes (genetic markers) are conditionally independent given the clustering (partition into subpopulations). We are not aware of any implementations under more complex models, for which the benefits of the approach should be more substantial. We stress that, unlike Gibbs sampling, HDS should not be viewed as a dual of EM; in Gibbs sampling and EM one only needs means for handling the conditional distribution of the hidden data $\mathbf{z}$ given the model parameters $\theta$ (either for sampling $\mathbf{z}$ or for computing suitable expectations), whereas HDS requires that one is able to compute the marginal probability of any given $\mathbf{z}$, obtained by integrating $\theta$ out.

We apply the HDS method for data clustering via mixtures of trees. We extend the mixture model of Meilă and Jordan [1] to a full Bayesian model and handle each mixture component using the algorithm of Meilă and Jaakkola [1]. We describe the building blocks in Sect. 2-4. In Sect. 5 we report experimental results on synthetic data, with a comparison to an EM algorithm. In Sect. 6 we apply the method to study how much Single Nucleotide Polymorphisms (SNPs) carry information about human subpopulations [12].

\section{Bayesian Networks and Trees}

A Bayesian network $(\mathrm{BN})$ over a vector of variables $x=\left(x_{1}, \ldots, x_{n}\right)$ specifies a probability distribution of $x$. The network structure of a BN encodes conditional independence assertions among the variables via a directed acyclic graph. We represent this graph as a vector $G=\left(G_{1}, \ldots, G_{n}\right)$ where each $G_{v}$ is a subset of the index set $V=\{1, \ldots, n\}$ and specifies the parents of $v$ in the graph. We may index with subsets: if $S=\left\{u_{1}, \ldots, u_{s}\right\}$ with $u_{1}<\cdots<u_{s}$, then $x_{S}$ denotes the vector $\left(x_{u_{1}}, \ldots, x_{u_{s}}\right)$. Along the structure $G$, a BN factorizes the probability 
distribution of $x$ into a product of local conditional distributions. Usually these conditional distributions belong to some parametric family, parametrized by $\theta$, and it is convenient to write the probability distribution of $x$ as

$$
p(x \mid G, \theta)=\prod_{v=1}^{n} p\left(x_{v} \mid x_{G_{v}}, G, \theta\right) .
$$

Our notation supports the Bayesian treatment of the network structure $G$ and the parameters $\theta$ as random variables (whenever their values are unknown).

A Bayesian tree (BT) is a Bayesian network where the network structure is a directed tree, i.e., one variable (the root) has no parents and every other variable has exactly one parent. BTs form an attractive subclass of BNs, as many important computational problems can be efficiently solved for trees but not for unconstrained BNs. We will soon come back to this issue.

BNs can be used to model multiple vectors $x[1], \ldots, x[m]$, called data and denoted by $\mathbf{x}$. When the vectors are judged to be exchangeable, the probability distribution of the data given the structure $G$, is expressed as

$$
p(\mathbf{x} \mid G)=\int\left(\prod_{j=1}^{m} p(x[j] \mid G, \theta)\right) p(\theta \mid G) \mathrm{d} \theta,
$$

where $p(\theta \mid G)$ is a parameter prior, and each $p(x[j] \mid G, \theta)$ factorizes as in (1). When the network structure is unknown, a prior distribution $p(G)$ is introduced and the marginal distribution of data can be written as

$$
p(\mathbf{x})=\sum_{G} p(G) p(\mathbf{x} \mid G),
$$

where $G$ runs through all possible network structures.

To best exploit the structural nature of BNs, usually the joint prior over structures and parameters is factorized by $p(G) \propto \prod_{v=1}^{n} \rho_{v}\left(G_{v}\right)$ and $p(\theta \mid G)=\prod_{v=1}^{n}$ $p\left(\theta_{v, G_{v}} \mid G_{v}\right)$, where each $\rho_{v}$ is a nonnegative function and $\theta_{v, G_{v}}$ is a set of parameters that fully specify the conditional distribution of $x_{v}$ given $x_{G_{v}}$. If a prior satisfies these conditions we call it modular or decomposable [131415]. Sometimes it also reasonable to force the prior to be likelihood equivalent 131415 , i.e., if two structures $G$ and $G^{\prime}$ represent the same assertions of conditional independence, then $p(G \mid \mathbf{x})$ and $p\left(G^{\prime} \mid \mathbf{x}\right)$ must be equal (for all data sets).

In this paper we consider the common setting of discrete variables with independent local multinomial distributions. We let the multinomial parameters be independent and follow a Dirichlet distribution such that both modularity and likelihood equivalence hold [1311.

A modular model structure makes computations easier, albeit not always feasible. In general, it is hard to find a network structure that maximizes the posterior $p(G \mid \mathbf{x})$ (see, e.g., 13 ), and we suspect that it is not easier to evaluate the probability of the data, $p(\mathbf{x})$. Recently, Koivisto and Sood 15 present an algorithm that solves these problems in time that scales as $n 2^{n}$. This algorithm is practical for small instances, up to about $n=25$ variables. 
On the contrary, for Bayesian trees several key tasks, such as maximum likelihood estimation and Bayesian inference, are computationally feasible even for large numbers of variables [16131. Meilă and Jaakkola 11] show the important result that also the marginal probability $p(\mathbf{x})$ for given data $\mathbf{x}$ can be computed in time cubic in $n$. The result applies whenever the structure prior is modular and symmetric, $\rho_{v}(u)=\rho_{u}(v) \geq 0$ for each edge $(u, v)$, and the parameters have a Dirichlet prior with the parameters (pseudo counts) $N_{u v}^{\prime}(s t)>0$, where $s$ and $t$ refer to the states of the variables $x_{u}$ and $x_{v}$, respectively; the counts $N_{u v}^{\prime}(s t)$ are subject to certain marginal constraints to ensure likelihood equivalence, see [17, Theorem 5] for details. The algorithm arises from an extension to the matrix tree theorem by Kirchhoff (in 1848); it computes the determinant of an $(n-1) \times(n-1)$ matrix that, in essence, represents for each pair of variables (excluding an arbitrarily chosen root) the marginal probability of the data on these variables. These probabilities are fully determined by the pairwise sufficient statistics, counts of occurrences for each value combination of two variables.

Unfortunately, the involved determinants tend to be ill-conditioned: to evaluate the determinant accurately one should let the precision of intermediate results grow linearly in the number of data points [18. Although this costs "only" about a linear factor in the time complexity [19, the algorithm seems to be practical for data sets with at most some thousands of records.

\section{Mixture Models and Clusterings}

Sometimes an inhomogeneous population can be well modeled by a relatively small number of homogeneous subpopulations. Finite mixture models embody this idea by forming the distribution of $x$ as a convex combination of a fixed number of other distributions, usually members of some parametric family. A mixture model with $k$ components is parametrized by mixture proportions $\alpha=\left(\alpha_{1}, \ldots, \alpha_{k}\right)$ which sum up to unity, and component-wise parameters $\beta=\left(\beta_{1}, \ldots, \beta_{k}\right)$, each $\beta_{c}$ specifying the $c$ th component distribution. Thus,

$$
p(x \mid \alpha, \beta)=\alpha_{1} f_{1}\left(x ; \beta_{1}\right)+\cdots+\alpha_{k} f_{k}\left(x ; \beta_{k}\right),
$$

where each $f_{c}\left(x ; \beta_{c}\right)$ belongs to a family of parametric distributions of $x$, which may be different for different components $c$. It is convenient to interpret a component as an unobserved variable $z$ that takes values in $\{1, \ldots, k\}$. Augmenting our probability model $p$ to $z$ we can write $\alpha_{c}=p(z=c \mid \alpha)$. Similarly we can replace $f_{c}\left(x ; \beta_{c}\right)$ by $p(x \mid \beta, z=c)$.

We now focus on mixtures of Bayesian networks and, in particular, of Bayesian trees. Thus, each $\beta_{c}$ specifies a network structure, $G_{c}$, and the parameters of the local conditional distributions, $\theta_{c}$. We can $\operatorname{read} p(x \mid \beta, z=c)$ as $p\left(x \mid G_{c}, \theta_{c}\right)$, which we expressed in (1).

The intimate relationship of mixture components and data clusters is obvious: for the $j$ th data point, the unobserved label $z[j]$ defines the cluster to which the data point belongs. Accordingly, the sets $\mathcal{C}_{c}=\{j: z[j]=c\}$, for $c=1, \ldots, k$, 
form a partition of the indices of the data points. To illustrate this relationship, we first write the distribution of the data $\mathbf{x}$ as

$$
p(\mathbf{x})=\iint\left(\prod_{j=1}^{m} p(x[j] \mid \alpha, \beta)\right) p(\alpha) p(\beta) \mathrm{d} \alpha \mathrm{d} \beta,
$$

where we assume that $\alpha$ and $\beta$ are independent a priori 1 Then we give an alternative expression as a sum over clusterings:

$$
p(\mathbf{x})=\sum_{\mathcal{C}} p\left(\mathcal{C}_{1}, \ldots, \mathcal{C}_{k}\right) p(\mathbf{x} \mid \mathcal{C})=\sum_{\mathbf{z}} p(\mathbf{z}) p(\mathbf{x} \mid \mathbf{z}),
$$

where $\mathbf{z}$ consists of the labels $z[j]$ and is one to one with $\mathcal{C}=\left(\mathcal{C}_{1}, \ldots, \mathcal{C}_{k}\right)$, and where the two terms in the latter sum have the following expressions. Assuming the prior of $\alpha$ is $\operatorname{Dirichlet}\left(m_{1}^{\prime}, \ldots, m_{k}^{\prime}\right)$, we have

$$
p(\mathbf{z})=\frac{\Gamma\left(m^{\prime}\right)}{\Gamma\left(m+m^{\prime}\right)} \prod_{c=1}^{k} \frac{\Gamma\left(m_{c}+m_{c}^{\prime}\right)}{\Gamma\left(m_{c}^{\prime}\right)},
$$

where $m_{c}=\left|\mathcal{C}_{c}\right|$ and $m^{\prime}=m_{1}^{\prime}+\cdots+m_{k}^{\prime}$. The second term can be written as

$$
p(\mathbf{x} \mid \mathbf{z})=\prod_{c=1}^{k}\left[\int\left(\prod_{j \in \mathcal{C}_{c}} p\left(x[j] \mid \beta_{c}, z[j]=c\right)\right) p\left(\beta_{c}\right) \mathrm{d} \beta_{c}\right]=\prod_{c=1}^{k} p\left(x\left[\mathcal{C}_{c}\right]\right),
$$

where $p\left(x\left[\mathcal{C}_{c}\right]\right)$ obeys (2), with $x\left[\mathcal{C}_{c}\right]$ denoting the data points in the $c$ th cluster.

We note that for a given clustering, the former term (3) is easy to evaluate, while computing the latter term (41) is feasible for general BNs on a small number of variables and for BTs on up to some hundreds of variables. This fact motivates our Markov chain sampler for the posterior distribution $p\left(\mathcal{C}_{1}, \ldots, \mathcal{C}_{k} \mid \mathbf{x}\right)=p(\mathbf{z} \mid \mathbf{x})$, which we describe in the next section 2

\section{MCMC over Clusterings}

We apply standard simulation techniques to draw a sample of clusterings along a Markov chain whose stationary distribution is the posterior distribution $p(\mathbf{z} \mid \mathbf{x})$. Posterior inference is then implemented via Monte Carlo averages.

\subsection{Sampling Along a Markov Chain}

We use the Metropolis-Hastings algorithm [20] with a simple proposal distribution as given as Algorithm 1, The algorithm is given as input a random initial clustering $\mathbf{z}^{(0)}$; in our experiments we used a very simple procedure that generates $k$ nonempty clusters of sizes $m_{1}, \ldots, m_{k} \geq 1$ with a probability proportional to the product $m_{1} m_{2} \cdots m_{k}$. The algorithm does not sample any tree structures or parameters. Instead we apply the results of Meilă and Jaakkola [1117] to calculate the exact average over all BTs as in (4) given a clustering.

\footnotetext{
${ }^{1}$ We slightly abuse the notation $\mathrm{d} \beta$, as $\beta$ contains also the network structure.

${ }^{2}$ We also remark that the prior $p(\mathbf{z})$ does not have to take the form (3) derived from the mixture model, but any prior, e.g., the uniform distribution, could be used.
} 


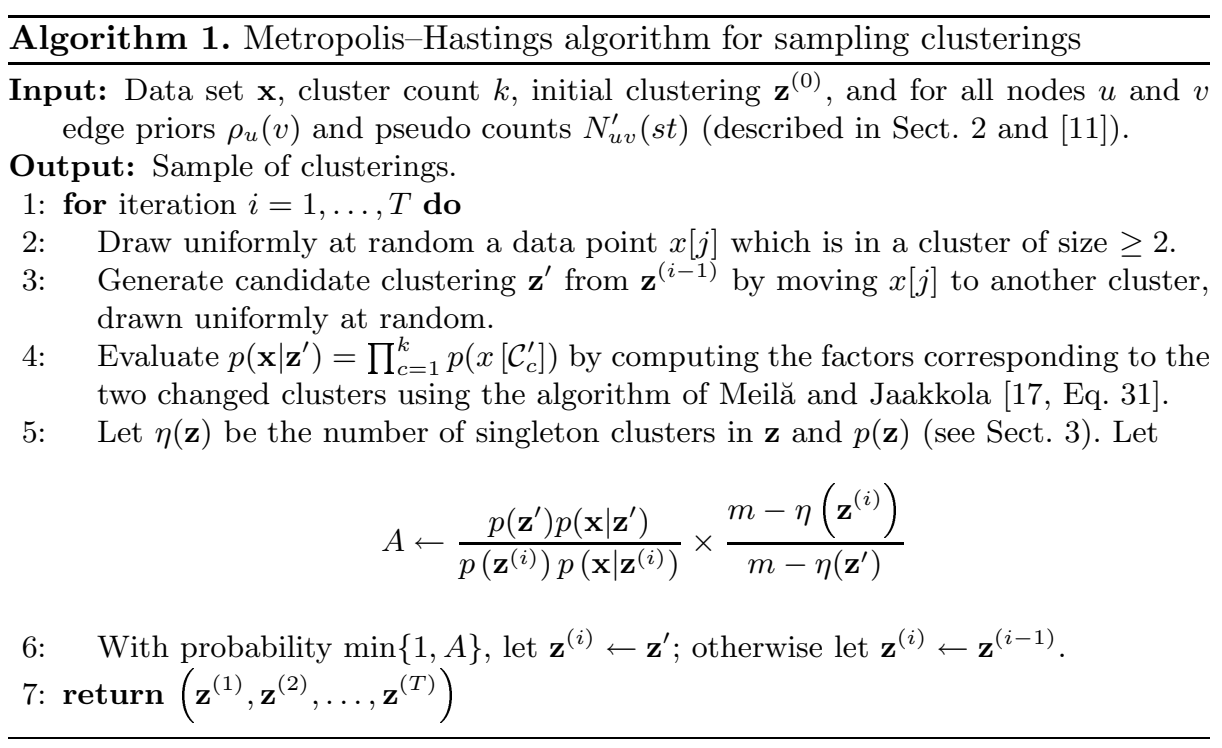

An advantage of this simple proposal distribution is that at each iteration only two data clusters are changed: a single data point is either removed or added. Thus, the $O\left(n^{2}\right)$ sufficient statistics needed for likelihood evaluation in tree structured models can be updated in $O\left(n^{2}\right)$ time, instead of the $O\left(m n^{2}\right)$ time needed for the initialization. In summary, the time complexity of Algorithm 1 is $O\left(m n^{2}+(k+T) n^{3}\right)$ where $T$ is the number of iterations and $n^{3}$ is the time required to evaluate the marginal probability of a single data cluster, given the sufficient statistics.

\subsection{Inference Using Monte Carlo}

The posterior expectation of any function $\phi(\mathbf{z})$ can be estimated by the arithmetic mean of the values $\phi\left(\mathbf{z}^{(i)}\right)$ at the sampled clusterings. For example, we may estimate the probability that two data points $j$ and $j^{\prime}$ belong to the same cluster by taking average of the indicator function $\mathrm{I}\left(z[j]=z\left[j^{\prime}\right]\right)$. While the posterior probabilities $p(z[j]=c \mid \mathbf{x})$ are rarely interesting 3 the pairwise counterparts, $p\left(z[j]=z\left[j^{\prime}\right] \mid \mathbf{x}\right)$, provide useful summaries, especially when the interest is in discovering data clusters; e.g., Dawson and Belkhir 9 apply a hierarchical clustering algorithm using the "co-assignment probabilities" as a measure of similarity.

We emphasize that one may also infer quantities that are indirect functions of clusterings. For example, we can estimate the posterior probability that all the $k$ trees include a particular edge $e$. To do this, we need to notice two facts. First, the events " $e$ belongs to the $c$ th tree" are mutually independent given

$\overline{{ }^{3} \text { For every } j}$ and $c$, the probability $p(z[j]=c \mid \mathbf{x})$ must equal $1 / k$, assuming that the prior is symmetric over the $k$ components. 


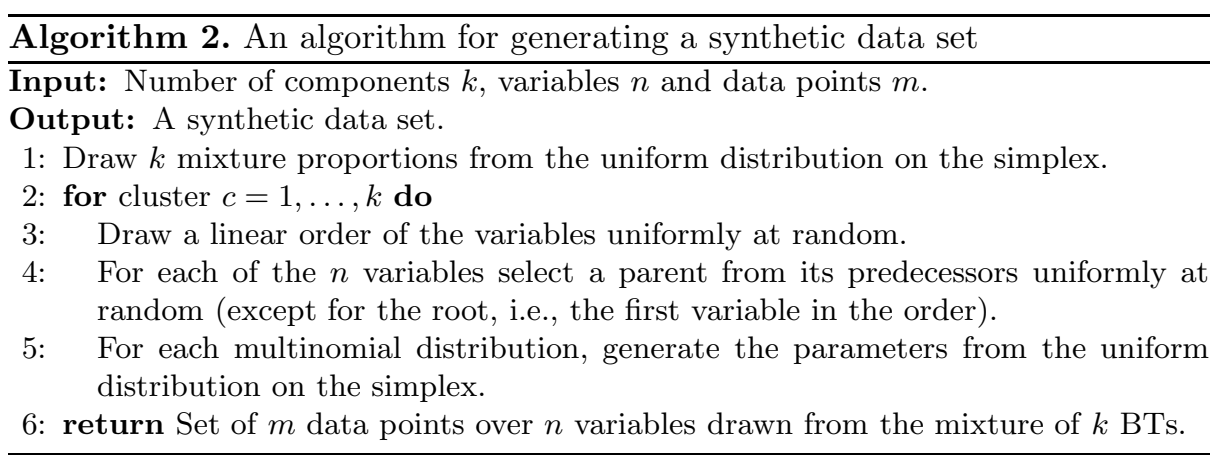

the clustering. Second, for each cluster we can efficiently compute the posterior probability that the corresponding tree includes the edge $e$ 11. Consequently, the probability that $e$ is included in every component is easy to compute for a given clustering. Taking a Monte Carlo average of these probabilities for the sampled clusterings finally yields the desired marginal posterior probability.

\section{Experimental Results on Synthetic Data}

We demonstrate the presented method in data clustering with mixtures of trees.

\subsection{The Clustering Problem}

We consider the following clustering problem. Given a set of data points, $x[1], \ldots, x[m]$, and a number $k$, the task is to assign a cluster label $z[j]$ from $\{1, \ldots, k\}$ to each data point $x[j]$ such that the global assignment, or clustering, $\mathbf{z}$ is as good as possible. We assume that there exists a unique correct clustering $\mathbf{z}^{*}$ and that the goodness of $\mathbf{z}$ is defined w.r.t. $\mathbf{z}^{*}$ via some discrepancy measure between two clusterings. Here we consider the Jaccard index [21], the ratio $a / b$, where $a$ is the number of pairs of data points that appear in the same cluster in both clusterings, and $b$ is the number of pairs of data points that appear in the same cluster in one or both clusterings. (We have also examined other measures, including the variation of information (VI) metric [22. The results are qualitatively very similar and therefore not reported here.)

\subsection{The Tested Methods and Data Sets}

We consider three scenarios of $(n, m, k)$ for the number of variables $n$, data points $m$, and clusters $k: \mathrm{A}=(10,100,2), \mathrm{B}=(20,200,4), \mathrm{C}=(50,500,10)$. For each scenario we generated 50 random data sets with two-state variables, and another 50 random data sets with four-state variables, as shown in Algorithm 2, Note that the described sampling distribution of trees is far from uniform: the flatter the tree, the larger the probability, chains being the least probable. 
In our experiments we used the following configuration of the proposed method. We set the prior over tree structures to the uniform distribution. Note that this distribution differs from the one we used in generating the test data sets. To the multinomial parameters and mixture proportions we assigned the uniform priors over the corresponding simplexes. In the determinant computations we used the standard double precision arithmetic, which is sufficient for our particular setup where every cluster typically contains less than 100 data points. As the posterior guess about the unobserved clustering we used the clustering that was visited by the simulated Markov chain and has the largest posterior probability. 4 We refer to this method as BMT (Bayesian mixture of trees). We assigned uniform prior over tree structures, i.e., equal $\rho_{u}(v)$ for each pair of nodes $(u, v)$. The parameter prior was set by $N_{u v}^{\prime}(s t) \equiv 1$; see Sect. 2 and Meilă and Jordan [17. for the interpretation of the parameters.

We compared BMT to our implementation of the MIXTREE algorithm [1] 5 This EM algorithm finds $k$ Bayesian trees along the mixture proportions so as to (locally) maximize the likelihood. We examined two initialization methods: either we generate a random mixture model, as done by Meilă and Jordan [1], or we draw a random soft clustering assignment, i.e., independent membership distributions for each data point; in the discussion below we will focus on the latter initialization method, for it produced slightly better results. Based on preliminary experiments we decided to stop each EM restart when the relative increase in the log-likelihood between the last two steps remains below the threshold $10^{-9}$ for two consecutive iterations; results for different stopping criteria were qualitatively very similar. Finally, a maximum likelihood clustering is found by assigning each data point to the most probable cluster, given the estimated mixture model and the data point. We refer to this method as MT (mixture of trees). It should be noted that we did not implement any of the smoothing tricks proposed by Meilă and Jordan [1, as they seem to lack a principled interpretation. Meilă and Jordan [1] report good results for MIXTREE in density estimation and classification; however, they do not consider the clustering task.

Both methods, BMT and MT, were given roughly the same amount of running time per data set. On each data set from scenarios A, B, and C, we allowed the algorithms run for 1,2 , and 5 hours, respectively. Given this amount of time, BMT typically completed millions of iterations, while MT completed thousands of random restarts of the EM algorithm.

\subsection{Analysis of Clustering Results}

The clustering results for BMT and MT are summarized in Fig. 1, On the smallest data sets (scenario A), BMT and MT perform about equally well on average.

\footnotetext{
${ }^{4}$ Alternatively, one could think of returning a clustering that maximizes the expected Jaccard index. However, such a clustering might be suboptimal w.r.t. other measures of clustering accuracy.

${ }^{5}$ We have implemented the algorithms in the $\mathrm{C}++$ language. The experiments were run on several $\mathrm{PCs}$, each having a $3.0 \mathrm{GHz}$ processor.
} 


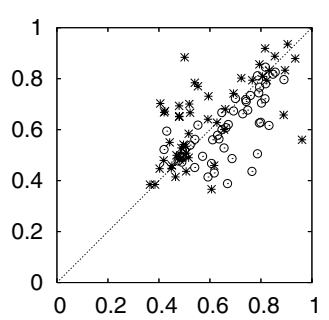

(a) Small data sets

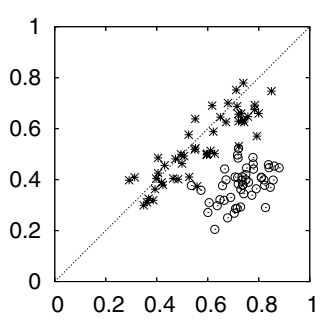

(b) Medium data sets

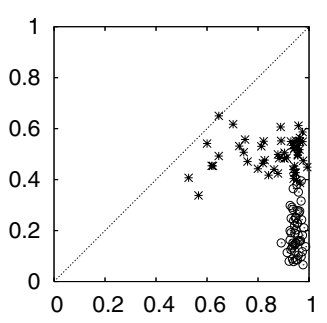

(c) Large data sets

Fig. 1. Clustering error measured by the Jaccard index, for BMT (x-axis) and MT ( $y$-axis). On scenarios A, B, and $\mathrm{C}$ (left to right) results are shown for 100 random data sets. The number of states per variable is two $(*)$ or four $(\odot)$.

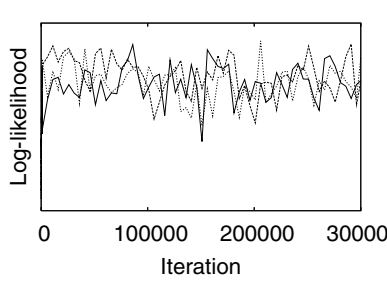

(a) A small data set

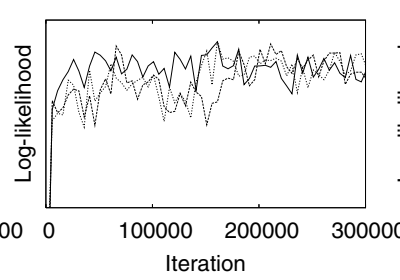

(b) A medium data set

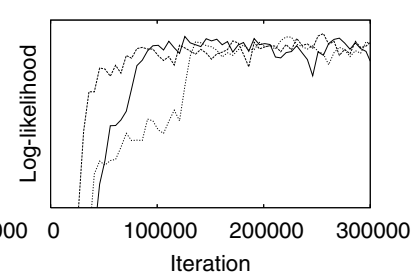

(c) A large data set

Fig. 2. Traces of the log-likelihood for three independent runs per scenario. Each picture shows every 5,000th sample from the first 300,000 iterations of three runs per case.

However, MT is slightly more accurate when the variables are binary-valued, whereas BMT is superior to MT when each variable can take four values. On the largest data sets (scenario C), BMT clearly outperforms MT. As expected, the difference in the performance grows with the number of states per variable.

We could explain these results theoretically as follows. When there are only 10 binary variables MT does not suffer much from using a point estimate (a mixture of BTs). On the contrary, BMT uses a somewhat biased prior, which renders its performance suboptimal. For larger numbers of variables the point estimates used by MT are no longer reliable - model averaging, as carried out by BMT, starts paying off. In practice, however, we realized that on larger data sets MT usually did not find the global optimum, despite the fair number of EM restarts. This may be the dominating reason for the relatively poor performance of MT. On the contrary, stochastic local search in the space of clusterings, as implemented in BMT, seems to be sufficient for finding plausible clusterings.

We visually inspected the mixing properties of BMT by running several independent chains from random initial states, for a few data sets selected at random. As shown in Fig. 2, mixing is rapid on the small data set, and, as expected, gets slower on larger data sets. Yet, the needed burn-in period is short compared to the total number of iterations for these data sets. 


\section{Application to SNP-Haplotype Clustering}

Geographically distant human subpopulations also tend to be genetically distant, relative to the variation within subpopulations. This is because after separation from a common founder population (a long time ago), subpopulations have evolved quite independently under population genetic forces, such as mutation, recombination, random drift, and selection. The variation within and between subpopulations can be observed at marker locations, especially, at Single Nucleotide Polymorphisms (SNPs). A SNP is a one-base location of the DNA where two different variants (alleles) appear in the population. Recently, large efforts have been put into typing (reading) millions of SNPs over the whole genome for groups of ethnically diverse individuals [12].

We apply the presented clustering method to study how many and how sparsely spaced SNPs are needed for inferring the known subpopulations solely from the SNP data. This question is interesting, since SNPs spanning a short region of the genome are not expected to be informative about the present subpopulations - rather, they reveal the content of the common, ancient founder population. We use the Perlegen data 12 that contains alleles at over 1.5 million SNPs for 24 European American samples, 23 African American samples, and 24 Han Chinese samples, each sample contributing two haplotypes to constitute a data set of 142 data points in total. Modeling with a mixture of trees makes a compromise with computational convenience and biological plausibility. Roughly speaking, we expect that the dependency of two markers gradually decays with their physical distance in the genome, suggesting a Markov chain model. However, several factors disturb this view, suggesting more complex models [23]. We use the BMT and MT methods with the details described in the previous section.

Figure 3 shows the results obtained at several randomly picked regions with varying number and spacing of the SNPs. We observe that the cluster structure of the data approaches the division into the three subpopulations as the number of SNPs and the average spacing get larger. For 100 SNPs with the average spacing of $500 \mathrm{~kb}$, the subpopulations are fairly accurately discovered by BMT. (The maximum-likelihood method, MT, is inadequate for revealing this trend.)

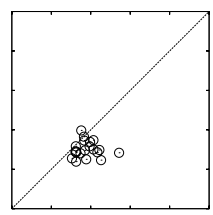

(a) $100,20 \mathrm{~kb}$

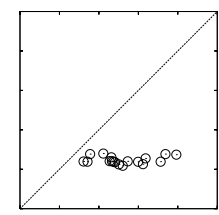

(b) $100,100 \mathrm{~kb}$

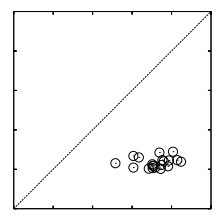

(c) $100,500 \mathrm{~kb}$

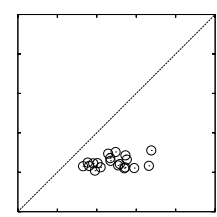

(d) $50,500 \mathrm{~kb}$

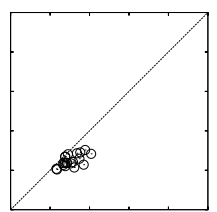

(e) $20,500 \mathrm{~kb}$

Fig. 3. Deviation of the subpopulation division and inferred clusterings measured by the Jaccard index, for BMT ( $x$-axis) and MT ( $y$-axis). Results shown for 20 random regions per case: $(\mathrm{a}-\mathrm{c}) 100 \mathrm{SNPs}$, the mean spacing of 20,100 , and $500 \mathrm{~kb}$, and (c-e) 100,50 , and 20 SNPs, the mean spacing of $500 \mathrm{~kb}$. The range of both axes is $[0,1]$. 


\section{Concluding Remarks}

We have applied the hidden data sampling (HDS) method for Bayesian learning with mixtures of graphical models. The HDS method is applicable whenever the likelihood of a single data cluster can be efficiently evaluated. We used Bayesian trees as an example of such a model class; Bayesian networks with a fixed variable order and bounded indegree is another example not considered here. Previous applications of essentially the same MCMC method have considered only simple Gaussian and product multinomial models 7/8910]. These works also show (rather straightforward) ways to extend the models and methods to an unknown number of clusters; notice, however, that inferring the number of clusters is usually quite sensitive to the priors of the model parameters.

In our experiments on data clustering, the proposed Bayesian method, BMT, outperformed the maximum likelihood method implemented via the EM algorithm, MT (MIXTREE, [1]). The observed poor performance of the EM algorithm is partially due to its sensitivity to the initial model. Another, and perhaps more interesting, reason is that MT fits all model parameters, including the tree structure, to a relatively small number of data points. To overcome this drawback, Meilă and Jordan [1] propose semi-Bayesian smoothing techniques to be used for small data sets. Our results suggest that "smoothing" is good to implemented in its entirety, via full model averaging, like done in BMT.

The results on the genetic data agreed with our hypothesis about the relationship of SNP haplotypes and human subpopulations. We note that, in general, population structure can be more powerfully inferred using data on different kind of genetic markers (microsatellites) 910. However, SNPs provide unique means for mapping disease predisposing genes [12] - it is useful to characterize what information SNPs carry about the genetic variation between individuals. Finally, we admit that trees are not perfect models for weakly correlated SNPs; in fact, independence models and, e.g., the k-means algorithm might perform equally well.

\section{Acknowledgments}

The authors would like to thank Heikki Mannila for useful discussions and Marina Meilă for providing some details of the MIXTREE algorithm.

\section{References}

1. Meilă, M., Jordan, M.I.: Learning with mixtures of trees. Journal of Machine Learning Research 1 (2000) 1-48

2. Efron, B.: Bootstrap methods: another look at the jackknife. Annals of Statistics 7 (1979) $1-26$

3. Alfaro, M.E., Zoller, S., Lutzoni, F.: Bayes or bootstrap? A simulation study comparing the performance of Bayesian Markov chain Monte Carlo sampling and bootstrapping in assessing phylogenetic confidence. Molecular Biology and Evolution 20 (2003) 255-266 
4. Diebolt, J., Robert, C.P.: Estimation of finite mixture distributions through Bayesian sampling. J. Royal Statistical Society B 56 (1994) 363-375

5. Jasra, A., Holmes, C.C., Stephens, D.A.: Markov chain Monte Carlo methods and the label switching problem in Bayesian mixture modelling. Statistical Science 20 (2005) 50-67

6. Celeux, G., Hurn, M., Robert, C.P.: Computational and inferential difficulties with mixture posterior distributions. J. Amer. Statist. Assoc. 95 (2000) 957-970

7. Neal, R.M.: Markov chain sampling method for Dirichlet process mixture models. Journal of Computational and Graphical Statistics 9 (2000) 249-265

8. Rasmussen, C.E.: The Infinite Gaussian Mixture Model. In Solla, S.A., Leen, T.K., Müller, K.R., eds.: NIPS 12, The MIT Press (2000) 554-560

9. Dawson, K.J., Belkhir, K.: A Bayesian approach to the identification of panmictic population and the assignment of individuals. Genetical Research 78 (2001) 59-77

10. Corander, J., Waldman, P., Sillanpää, M.J.: Bayesian analysis of genetic differentiation between populations. Genetics 163 (2003) 367-374

11. Meila, M., Jaakkola, T.: Tractable Bayesian learning of tree belief networks. In Boutilier, C., Goldszmidt, M., eds.: UAI, Morgan Kaufmann (2000) 380-388

12. Hinds, D.A., et al.: Whole-genome patterns of common DNA variation in three human populations. Science 307 (2005) 1072-1079

13. Heckerman, D., Geiger, D., Chickering, D.M.: Learning Bayesian networks: The combination of knowledge and statistical data. Machine Learning 20 (1995) 197243

14. Friedman, N., Koller, D.: Being Bayesian about network structure: A Bayesian approach to structure discovery in Bayesian networks. Machine Learning 50 (2003) 95-125

15. Koivisto, M., Sood, K.: Exact Bayesian structure discovery in Bayesian networks. Journal of Machine Learning Research 5 (2004) 549-573

16. Chow, C., Liu, C.: Approximating discrete probability distributions with dependence trees. IEEE Transactions on Information Theory 14 (1968) 462-467

17. Meilă, M., Jaakkola, T.: Tractable Bayesian learning of tree belief networks. Technical Report CMU-RI-TR-00-15, Carnegie Mellon University Robotics Institute (2000)

18. Cerquides, J., de Mántaras, R.L.: TAN classifiers based on decomposable distributions. Machine Learning 59 (2005) 1-32

19. Kaltofen, E., Villard, G.: On the complexity of computing determinants. Computational Complexity 13 (2004) 91-130

20. Hastings, W.K.: Monte Carlo sampling methods using Markov chains and their applications. Biometrika 57 (1970) 97-109

21. Jaccard, P.: The distribution of the flora in the Alpine zone. The New Phytologist XI (1912) 37-50

22. Meila, M.: Comparing clusterings by the variation of information. In Schölkopf, B., Warmuth, M.K., eds.: COLT. Volume 2777 of Lecture Notes in Computer Science., Springer (2003) 173-187

23. Thomas, A., Camp, N.J.: Graphical modeling of the joint distribution of alleles at associated loci. American Journal of Human Genetics 74 (2004) 1088-1101 